\title{
Suitability of PLLA as piezoelectric substrates for tissue engineering evidenced by microscopy techniques
}

\author{
N. B. Barroca*, A. L. Daniel-da-Silva*, P. S. Gomes**, M. H. R. Fernandes**, S. Lanceros- \\ Méndez $^{* * *}$, P. Sharma ${ }^{* * *}$, A. Gruverman****, M. H. V. Fernandes* and P. M.Vilarinho* \\ * CICECO, Dep. of Ceramics and Glass Engineering, University of Aveiro, Campus de Santiago, \\ 3810-193 Aveiro, Portugal \\ ** Faculty of Dental Medicine, University of Porto, 4200-393 Porto, Portugal \\ *** Department of Physics, University of Minho, Campus de Gualtar, 4710-057, Braga, Portugal \\ **** Department of Physics and Astronomy, University of Nebraska-Lincoln, 202 Ferguson Hall, \\ Lincoln, NE 68588-0111, USA
}

Since the discovery of the piezoelectric character of bone, the suitability of some piezoelectric materials have been studied for bone repair; they are thought to act like transducers converting the mechanical energy of skeletal deformation in electrical stimuli capable of controlling osteogenic growth. The mechanisms underlying this process are far from being understood and systematic studies at a local scale are required. Atomic force microscopy (AFM) is a unique way to observe phenomena at the nanoscale and liquid imaging provides a unique tool to assess biological phenomena at the nanoscale. So in this study, aiming at a better understanding of the role of piezoelectricity in the osteogenic growth, the interaction between a poled piezoelectric material, in this case poly (L-lactic) acid and an adhesion promoting protein, the fibronectin, and bone-like cells is evaluated by scanning probe microscopy and confocal laser scanning microscopy (CLSM).

Poly (L-lactic) acid (PLLA), a semi-crystalline synthetic polymer, is a promising material for bone tissue engineering due to its particular properties combination: biodegradability with biocompatibility and piezoelectricity. Hence, PLLA has been the object of our current studies.

Two different kinds of poled PLLA samples to be used as piezoelectric substrates for bone tissue engineering were prepared. The first set was substrate free, obtained by solvent-casting and macroscopically poled by corona poling. The second set was prepared by spin-coating on $\mathrm{Pt} / \mathrm{TiO}_{2} / \mathrm{SiO}_{2} / \mathrm{Si}$ substrates and poled locally by applying a DC field through a piezoresponse force microscopy (PFM) tip. Field induced molecular orientation in PLLA was imaged by PFM. Poled and non-poled PLLA films were afterward brought into contact with human serum fibronectin, MG-63 osteoblast-like bone cells and human bone marrow cells.

Atomic force microscopy in tapping mode and in liquid environment was used to observe the effect of the substrate polarization on the protein adhesion. Atomic force microscopy liquid imaging provides an environment for the proteins closer to their ones and avoids denaturation. Tapping mode allows imaging proteins without damaging them. Acquired data showed a significant higher quantity of proteins on the poled area of PLLA (Figure 1) [1]. Molecular force spectroscopy was then used to check if proteins are adsorbed on the surface. Indeed force curves displayed multiple rupture events characteristic of adhesion events between AFM tip and adsorbed proteins.

Confocal laser scanning microscopy (CLSM) combined with MTT and alkaline phosphatase (ALP) assays depicted the ability of all prepared films to support adhesion, spreading and proliferation of bone-like cells. Particularly, the negatively poled PLLA substrates showed a higher adhesion and proliferation of cells (Figure 2).

In summary, different microscopy techniques were combined in this study to investigate the effect of poled surface on adhesion-promoting proteins and bone-like cells. The polarization of PLLA resulted in enhanced protein adsorption and also in an evident effect regarding cells behaviour: the negatively poled PLLA enhanced cells adhesion, spreading and proliferation, particularly at the early stage comparing with non-poled or positively poled. 


\section{References}

1. Barroca et al., Applied Physics Letters 98: 133705, 2011
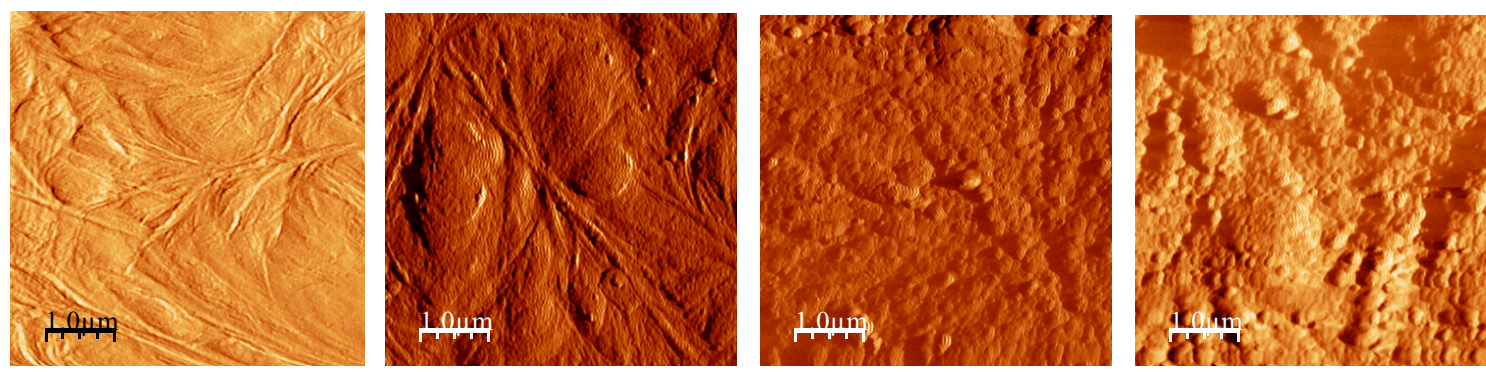

Figure 1. Topography images of PLLA thin film surfaces acquired in AFM tapping mode in liquid: (a) non poled area before protein adsorption; (b) non-poled area after protein adsorption; (c) positively poled area after protein adsorption; (d) negatively poled area after protein adsorption.
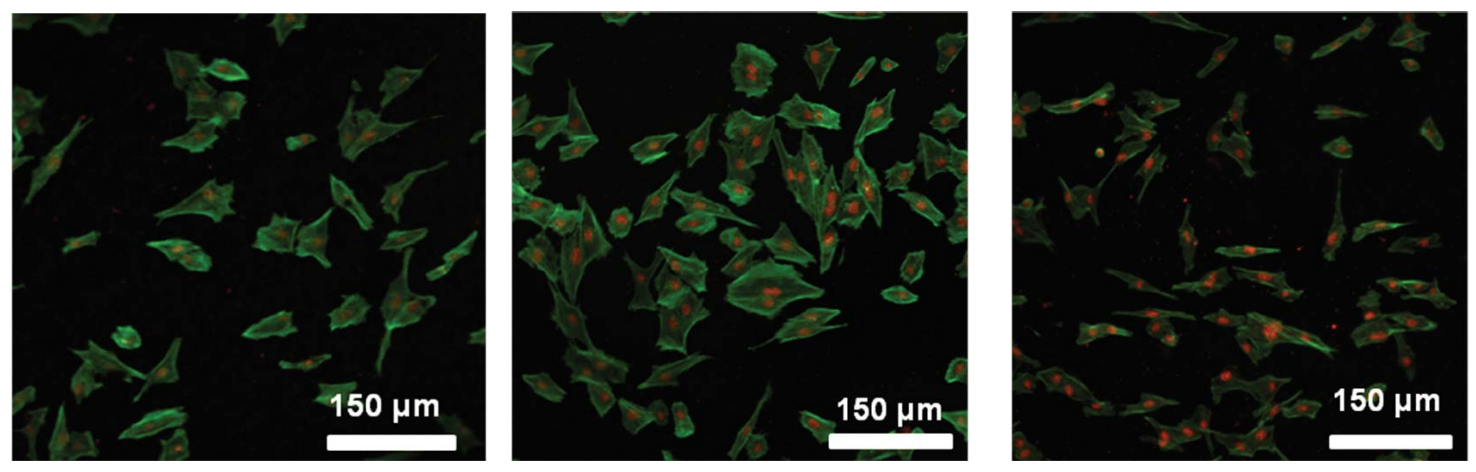

Figure 2. CLSM micrographs of cultures stained for F-actin cytoskeleton (green) and nucleus (red). Proliferation of MG63 osteoblast-like cells over PLLA films, pre-coated with fibronectin: (a) nonpoled, (b) negatively charged and (c) positively charged PLLA $(+)$. 